ARTICLE

\title{
The TatD-like DNase of Plasmodium is a virulence factor and a potential malaria vaccine candidate
}

Zhiguang Chang ${ }^{1}$ Ning Jiang ${ }^{1}$, Yuanyuan Zhang ${ }^{1}$, Huijun Lu¹, Jigang Yin ${ }^{1}$, Mats Wahlgren², Xunjia Cheng ${ }^{3}$, Yaming $\mathrm{CaO}^{4} \&$ Qijun Chen ${ }^{1,2,5}$

Neutrophil extracellular traps (NETs), composed primarily of DNA and proteases, are released from activated neutrophils and contribute to the innate immune response by capturing pathogens. Plasmodium falciparum, the causative agent of severe malaria, thrives in its host by counteracting immune elimination. Here, we report the discovery of a novel virulence factor of $P$. falciparum, a TatD-like DNase (PfTatD) that is expressed primarily in the asexual blood stage and is likely utilized by the parasite to counteract NETs. PfTatD exhibits typical deoxyribonuclease activity, and its expression is higher in virulent parasites than in avirulent parasites. A P. berghei TatD-knockout parasite displays reduced pathogenicity in mice. Mice immunized with recombinant TatD exhibit increased immunity against lethal challenge. Our results suggest that the TatD-like DNase is an essential factor for the survival of malarial parasites in the host and is a potential malaria vaccine candidate.

\footnotetext{
${ }^{1}$ Key Laboratory of Zoonosis, Jilin University, Xi An Da Lu 5333, Changchun 130062, China. ${ }^{2}$ Institute of Microbiology, Tumour and Cellular Biology, Karolinska Institutet, Nobels väg 16, S-171 77 Stockholm, Sweden. ${ }^{3}$ Department of Pathogen Biology, Fudan University, Handan Road 220, Shanghai 200433, China. ${ }^{4}$ Department of Immunology, China Medical University, Puhe Road 77, Shenyang 110122, China. ${ }^{5}$ Key Laboratory of Zoonosis, Shenyang Agricultural University, Dongling Road 120, Shenyang 10866, China. Correspondence and requests for materials should be addressed to Q.C. (email: caj@jlu.edu.cn or syaucaj@163.com).
} 
$\mathrm{n}$ activation by initial microbial contact, neutrophils and macrophages export filamentous elements composed of DNA and proteases, which confine the invading pathogens ${ }^{1,2}$. These filamentous structures are known as neutrophil extracellular traps (NETs) and have been demonstrated to contribute to pathogen capture as part of the innate immune response ${ }^{3,4}$. Correspondingly, some pathogens successfully escape the trap by secreting DNases to degrade the $\mathrm{DNA}^{5,6}$. The capacity of the released DNases to counteract NET confinement renders them microbial virulence factors ${ }^{7-10}$. Furthermore, plasma haem can induce the formation of NETs in vitro ${ }^{11}$, and haem is abundantly released in the host-blood circulation on the rupture of Plasmodium falciparum-infected erythrocytes ${ }^{12,13}$. NET structures were observed in the skin-associated peripheral blood of a $P$. falciparum-infected child with uncomplicated malaria ${ }^{14}$. $P$. falciparum, the causative agent of severe malaria, thrives in its host by counteracting immune elimination ${ }^{15-17}$

We hypothesize that NETs may be an important innate immune factor for controlling malarial parasite proliferation in the host, and the DNase that is released from the parasite may be a critical factor for NET destruction. In this study, we identify a DNase sequence in Plasmodium falciparum that contains a signal peptide and is homologous to bacterial TatD DNases (herein named TatD-like DNases) that are actively expressed in both $P$. falciparum and in rodent malarial parasites. The expression of this TatD-like DNase is associated with parasite virulence, and TatD-like DNase-specific antibodies are key elements in providing anti-plasmodium immunity.

\section{Results}

The plasmodial TatD possesses a conserved feature of DNases. Proteins of the TatD deoxyribonuclease family participate in many different biological processes, such as protein export and pathogenesis ${ }^{18,19}$. The sequence of $P$. falciparum (PfTatD) (PF3D7_0112000) is $\sim 30 \%$ similar to that of Homo sapiens, zebrafish, Escherichia coli and protozoa (Fig. 1a and Supplementary Fig. 1). The predicted PfTatD 3D model (without its signal peptide) contains a hydrolase active site surrounded by seven $\alpha$-helices (Fig. 1b). The PfTatD domain contains a TIM barrel (COG 1099, multiple domain), which represents a nucleic acid binding region ${ }^{20}$ (Fig. 1c; cerulean, rounded rectangle).

Furthermore, compared to E. coli TatD, Plasmodium TatD contains an insertion in the middle of the sequence consisting of 73 amino acids (AAs) (Fig. 1a-c; yellow box, yellow tape and yellow rounded rectangle, respectively), which marks another feature of the Plasmodium TatDs that is distinct from other microbial homologues, apart from the signal peptide.

The expression of TatD is associated with virulence. Transcription of PfTatD began early after parasite invasion into the erythrocytes, reaching the maximum level of transcription at $32 \mathrm{~h}$ and declining steeply at $40 \mathrm{~h}$ (Fig. 2a). The expression of the PfTatD protein began early after erythrocyte invasion and reached a maximal level $24 \mathrm{~h}$ post invasion, as shown by western blot analysis with a specific anti-rPfTatD antibody (Fig. 2b). Indirect immunofluorescence assays revealed that PfTatD was primarily localized to the periphery of infected erythrocytes at the mature trophozoite stage (Fig. 2c). Immunoelectron microscopy illustrated that PfTatD was primarily localized outside the parasitophorous membrane, with a trend towards localization at the red blood cell membrane (Fig. 2d-f). A similar pattern of expression was also observed in the infected red blood cells (iRBCs) of the Plasmodium berghei strain ANKA (Supplementary Fig. 2).
To determine whether expression of the plasmodial TatD-like DNase is associated with pathogenicity or parasite proliferation within the host, we compared the expression of homologous proteins in several rodent parasite strains with lethality in mice. Transcription and expression of the $P$. berghei TatD-like DNase (PbTatD) were quantitatively higher in the virulent ANKA strain of $P$. berghei than the avirulent strain NK65 (Fig. 3a,c). This trend was also observed in strains of the $P$. yoelii parasite; specifically, the more virulent YM strain expressed more TatD than the avirulent XNL strain (Fig. 3b,d). Thus, the TatD-like protein is predominantly expressed in virulent parasite strains.

In addition, gene knockout, complementation and mutation studies were performed to further confirm the hypothesis that TatD-like DNase is essential for parasite virulence. First, the TatD-like DNase gene of $P$. berghei ANKA was deleted using double-crossover method, a technique commonly applied in the functional analysis of malarial parasites ${ }^{21}$ (Supplementary Fig. 3a). The knockout strain $(\triangle \mathrm{PbTatD})$ was cloned and verified using both PCR with gene-specific primers and western blot assays (Supplementary Fig. 3b,c). The ability of the $\triangle \mathrm{PbTatD}$ strain to proliferate in vivo was diminished compared with that of the wild-type (WT) strain (Fig. 4a,b). Second, the gene encoding $\mathrm{PbTatD}$ was re-introduced into the $\triangle \mathrm{PbTatD}$ strain (named PbTatD-com), targeting the d-ssu-rrna gene with the selectable marker human dihydrofolate reductase (hDHFR) (Supplementary Fig. 4). Pathogenicity, in terms of proliferation rate and lethality, was fully restored in the knockout parasite after complementation with the PbTatD gene (Fig. 4a,b). Third, we introduced an aspartic acid-to-alanine mutation at the 352 position of $\mathrm{PbTatD}$ (named PbTatD-D352A) (Supplementary Fig. 5). The PbTatD-D352A mutation strain showed reduced proliferation and lethality in infected mice (Fig. 4a,b). Thus, the data collectively support our supposition that TatD-like DNase is a parasite virulence factor essential for the proliferation and pathogenicity of the parasite.

Plasmodial TatD is a divalent metal-independent DNase. It was previously shown that the TatD of E. coli possesses DNA hydrolase activity ${ }^{22}$. We further characterized the DNA hydrolase activity of PfTatD by incubating the recombinant protein rPfTatD-GST (Supplementary Fig. 6) with human DNA in the presence of various divalent ions. First, rPfTatD-GST was able to hydrolyze double-stranded DNA in a concentrationdependent manner (Supplementary Fig. 7a), with maximal activity observed at 37 and $41^{\circ} \mathrm{C}$ (Supplementary Fig. 8a). Such temperatures are not physiological but are commonly observed in infected patients. Because the activity of yeast and E. coli TatD is dependent on $\mathrm{Mg}^{2}+$ (refs 23,24), we investigated the effect of divalent ions on the enzymatic activity of plasmodial TatD. Unexpectedly, the DNA hydrolase activity of rPfTatD was increased in the presence of higher concentrations of EDTA in the reaction buffer and, conversely, was inhibited by the addition of $\mathrm{Mg}^{2+}, \mathrm{Ni}^{2+}$ or other metals ions (Supplementary Figs. $7 b, c$ and $8 b$ ). Furthermore, it has been reported that histidine and aspartic acid are critical for the structure and function of DNA hydrolases ${ }^{25}$. Four histidines and one aspartic acid residue were predicted in the sequences of plasmodial TatD hydrolases. To demonstrate that these two AAs are essential, we generated recombinant proteins containing mutations introduced at either the $\mathrm{H} 303$ or D352 position (Supplementary Fig. 5a) and tested their DNA hydrolytic activity. The results showed that none of these mutated proteins had DNase activity (Supplementary Fig. 5b,c). Thus, plasmodial TatD is a DNA deoxyribonuclease with a biochemical feature distinct from other DNA hydrolases. 
a

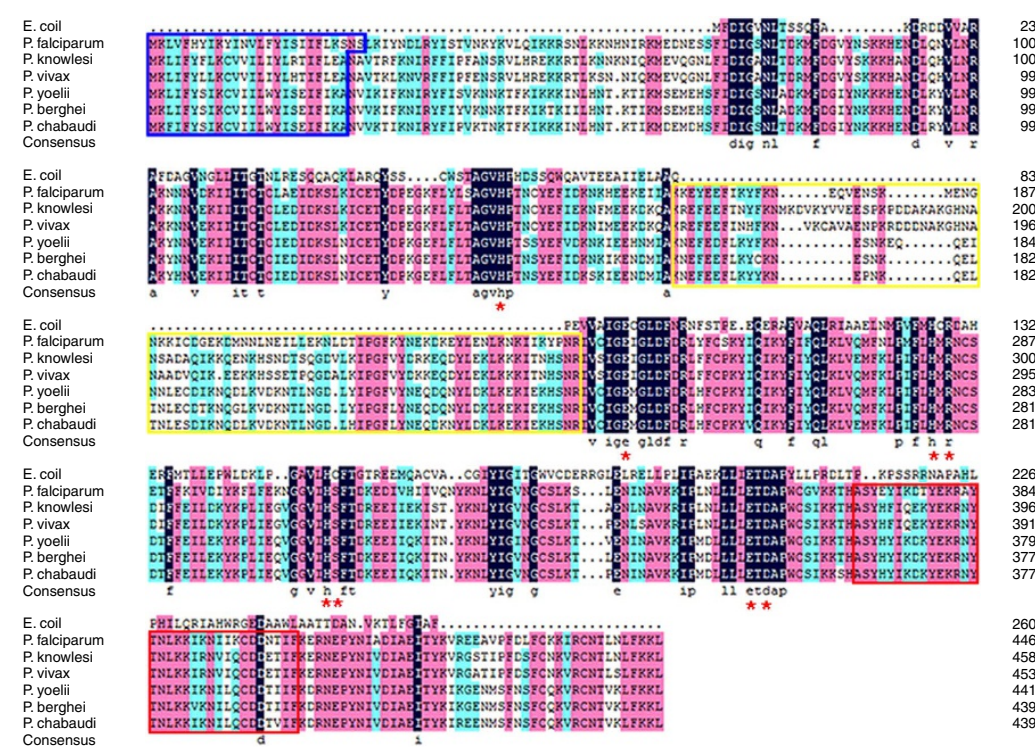

b

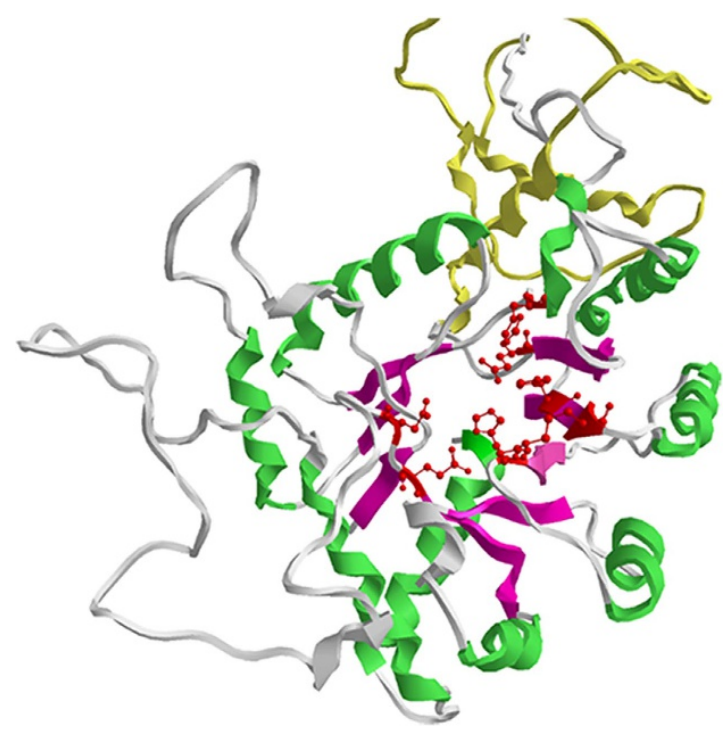

C

TatD DNase

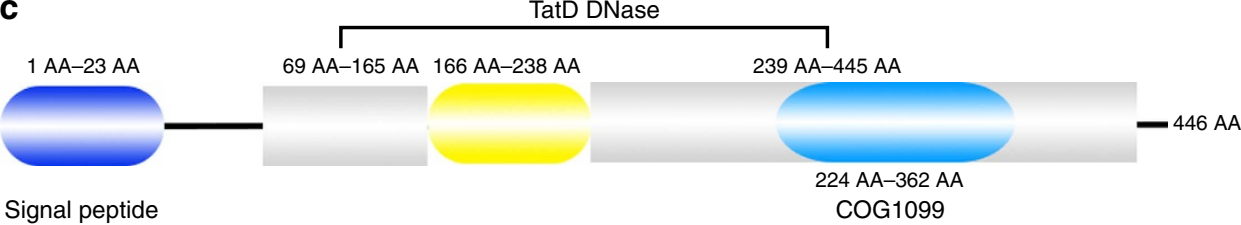

Figure 1 | Sequence analysis and the predicted structure of PfTatD. (a) The TatD sequence alignment across various strains of Plasmodium and $E$. coli. The plasmodial TatD sequences contain a 24-26 AA signal peptide, whose cleavage site was between residues 26 and 27 in the $P$. falciparum or between 24 and 25 in other genera (blue box). A gap appeared in the predicted TatD DNase domain (yellow box) following a potent cell-binding residue (red box). The AAs that constitute the active site are indicated by red stars along the sequence. (b) The PfTatD 3D structure model was built using SWISS-MODEL. A template that contained $9 \alpha$-helices (green bands) and $8 \beta$-pleated sheets (purple bands) in the secondary structure constituted a stable region. The yellow band indicates the gap in the TatD DNase domain. The red cylindrical bonds (red stars) indicate the conserved active site presented in a. (c) The schematic structural composition of the PfTatD domains. A 23-AA signal peptide site at the $\mathrm{N}$ terminus, followed by a 73-AA sequence gap (yellow rounded rectangle) that interrupted the TatD DNase domain, and AA residues from 224 to 362 indicated by the cerulean rounded rectangle.

Plasmodial TatD hydrolyzes macrophage-derived ET. The involvement of parasite-infected erythrocytes in the induction of extracelluar trap (ET) structures in vitro was first tested by co-cultivation of J774A.1 macrophages with RBCs that were infected with either WT $P$. berghei, the $\triangle \mathrm{PbTatD}$ strain, the PbTatD-D352A mutant strain or the PbTatD-com strain. Both the $\triangle \mathrm{PbTatD}$ and $\mathrm{PbTatD}-\mathrm{D} 352 \mathrm{~A}$ mutant strains induced ET network formation in vitro, as is commonly observed in bacterial pathogens after incubation with macrophages, but there were significantly fewer ET nets induced by RBCs infected with WT $P$. berghei and the PbTatD-com strain (Fig. 5a-c, $P<0.05$ in two-tailed Student's $t$-test). Similarly, mouse neutrophils could 
a

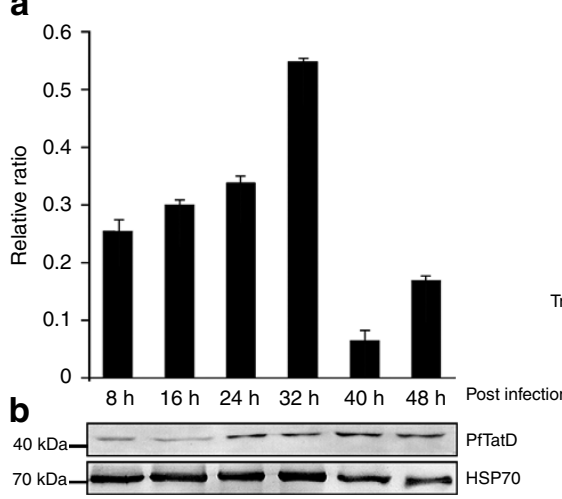

C

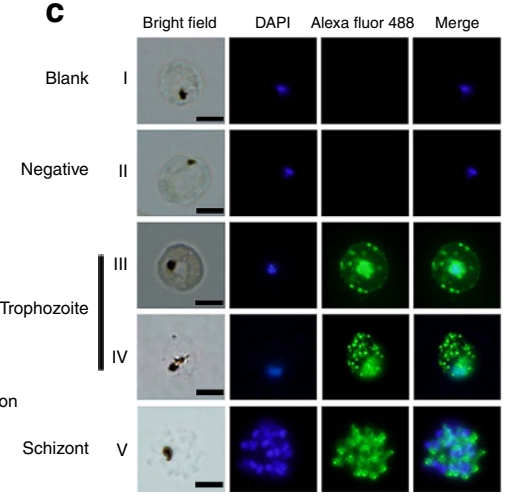

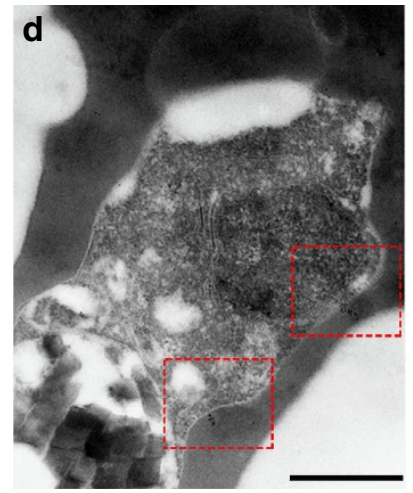

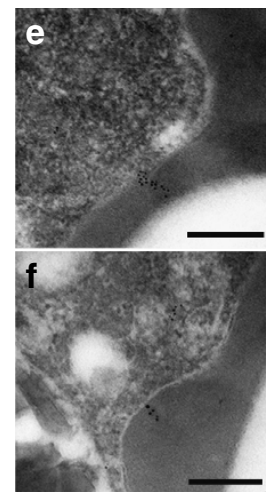

Figure 2 | PfTatD is expressed at the mature stage of trophozoite-infected erythrocytes. (a) Transcriptional analysis of the PF3D7_0112000 gene was performed using quantitative PCR. Transcription was increased with parasite growth and reached its maximum at $32 \mathrm{~h}$ in the blood stage. The results are shown as the mean \pm s.e.m. of 3 independent experiments. (b) An immunoblot was performed with rabbit anti-rPfTatD IgG (also see Supplementary Fig. 13). The top line indicates the expression of PfTatD every $8 \mathrm{~h}$ and the increased expression at $32 \mathrm{~h}$ in the blood stage. HSP70, presented in the bottom panel, was used as a control. (c) Indirect immunofluorescence of PfTatD was detected. The green fluorescence indicates that PfTatD was localized to the RBC membrane or the parasitophorous vacuole membrane of a mature trophozoite and was distinct from the nucleus in the schizont. The parasite nuclei were stained with DAPI. Scale bar, $5 \mu \mathrm{m}$. (d-f) Immune electron microscopy images of PfTatD. The gold particles were localized to the parasitophorous vacuole membrane, and there was a trend for localization to the periphery. Scale bar, (d) $500 \mathrm{~nm}$ and (e,f) $200 \mathrm{~nm}$.

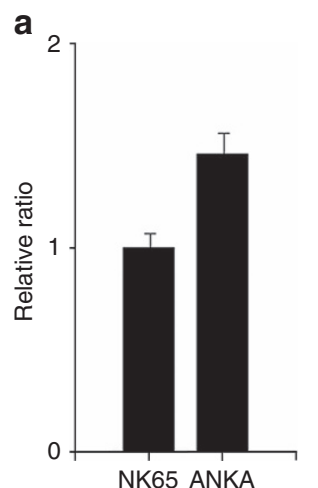

C

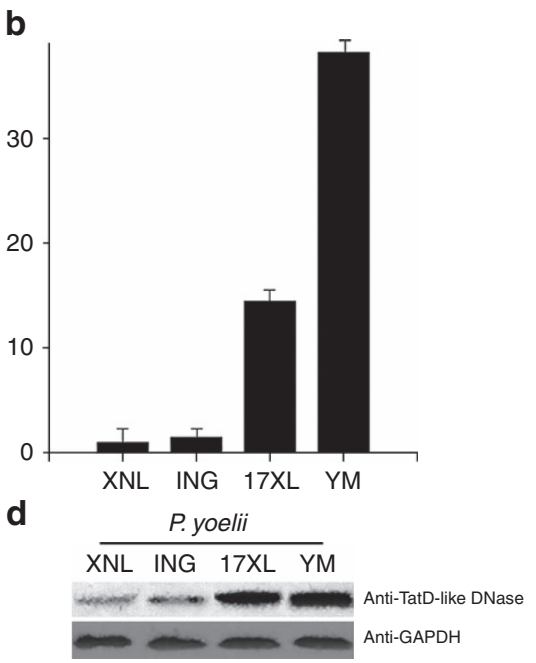

Figure 3 | TatD-like DNase expression is associated with parasite virulence. Quantitative PCR and western blot assays were performed to measure mRNA and protein levels, respectively, of the TatD-like DNase in rodent malarial parasites. In the histograms presented in $\mathbf{a}, \mathbf{b}$ parasite pathogenicity increased from the left to right, which positively correlated with transcription. Error bars represent the mean \pm s.e.m. of three independent experiments. The expression patterns presented in $\mathbf{c}, \mathbf{d}$ also positively correlated with the transcription results. The top panel indicates the TatD expression in different strains, which were collected at the same stage of the parasites. GAPDH is presented in the bottom panel and was used as a control. The full images of the western blot assays are shown in Supplementary Fig. 13.

generate ET when co-cultivated with the $\triangle \mathrm{PbTatD}$ strain, but they could not produce ET in the presence of WT parasites (Supplementary Fig. 9). Thus, WT P. berghei ANKA inhibits ET formation in a PbTatD-dependent manner, which supports our hypothesis that TatD-like DNase is released from parasite-infected RBCs and mediates the interaction of the parasite with the host innate immune system ${ }^{10,26}$.

To further confirm the role of the TatD enzyme in the interaction of plasmodial parasites with host immune cells, recombinant proteins of either $\mathrm{rPbTatD}-\mathrm{GST}$ or $\mathrm{rPbTatD}$ D352A-GST were added into $\triangle \mathrm{PbTatD}$ strain/macrophage cultures. Although rPbTatD-GST could hydrolyze ET structures in a time-dependent manner, the mutated protein showed no function in DNA hydrolysis at any time point in the assays (Fig. 5d,e). Thus, the data collectively support the hypothesis that plasmodial TatDs are parasite virulence factors that may be released from infected erythrocytes to hydrolyze the DNA component in ET structures formed by immune cells.

Immunization of recombinant TatD protects against infection. We further investigated the significance of TatD-specific antibodies in host protection against parasite infection in vivo. BALB/ c mice ( $N=15$ in each group) were immunized four times with the His-tagged recombinant $P$. berghei TatD-like DNase protein (rPbTatD-HIS) (Fig. 6a) and Freund's adjuvant. As expected, a reduction in parasitaemia and delayed death was observed in the immunized group after challenge by intraperitoneal (IP) injection with $10^{6}$ iRBCs (Fig. 6b,c). Furthermore, there was a reduction in parasitaemia and protection in infected mice passively treated with intravenous (IV) injection of anti-rPbTatD sera (Fig. 6f,g). Moreover, immunization with the His-tagged recombinant TatD-like DNase of $P$. chabaudi (rPcTatD-HIS) produced similar results (Fig. 6d,e,h,i). The experiment was repeated with C57BL/6 mice, and similar results were obtained (Supplementary Fig. 10). Cross species protection was tested. Mice were immunized with rPbTatD-HIS and challenged with either the P. chabaudi AS or $P$. yoelii 17XL strain. A reduction in parasitaemia and delayed death was observed in the immunized group (Supplementary Fig. 11). Moreover, plasmodial TatD-specific antibodies showed very minimal cross-reaction with host proteins of various organs (Supplementary Fig. 12). These data collectively indicate that TatD-specific antibodies provide protection against parasite infection.

\section{Discussion}

There are diverse mechanisms by which malaria parasites escape host innate immunity ${ }^{27}$, but the proteins involved in these processes are poorly understood. These molecules are likely to be important virulence factors that can be explored as potential candidates for vaccine development or novel therapies. 

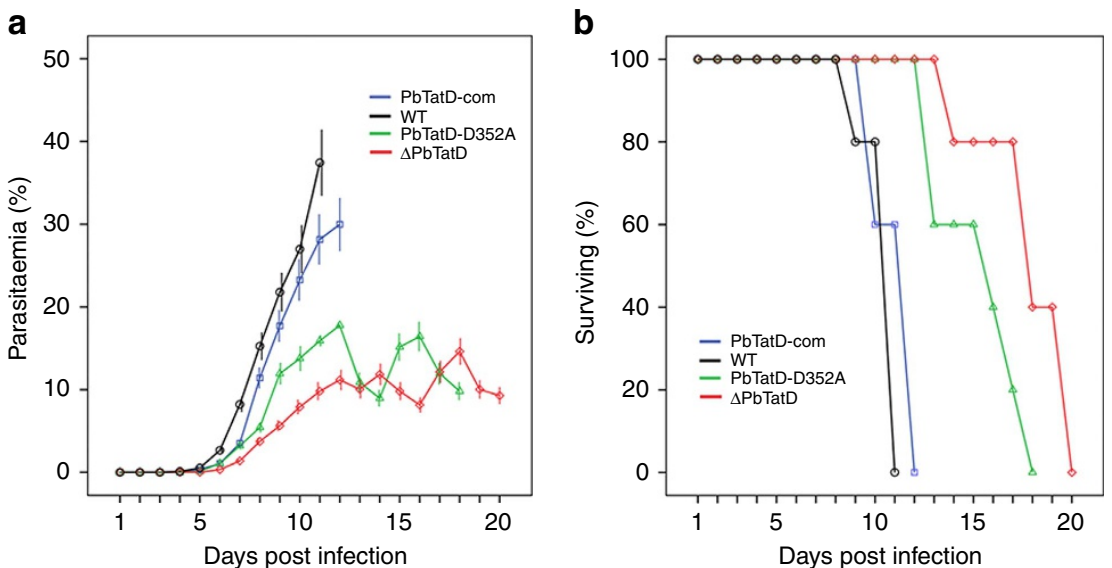

Figure 4 | A comparison of the parasitaemia and survival of mice infected with WT and mutated strains. (a) A comparison of the mean parasitaemia with standard variations of the mice that were infected with WT P. berghei, $\triangle \mathrm{PbTatD}, \mathrm{PbTatD}-\mathrm{D} 352 \mathrm{~A}$ and PbTatD-com parasites from day 1-20 after infection. The parasitaemia in mice that were infected with either $\triangle$ PbTatD (red line) or PbTatD-D352A (green line) was lower than in mice infected with WT (black line) or PbTatD-com parasites (blue line). The error bars indicate s.d. (b) The curves representing the survival rates of the mice that were infected with WT, $\triangle$ PbTatD, PbTatD-D352A and PbTatD-com strains. The mice that were infected with the WT exhibited $100 \%$ death 11 days after IP injection with $10^{6}$ infected RBCs. The PbTatD-com strain exhibited a similar result as WT, whereas the mice that were infected with the $\Delta \mathrm{PbTatD}$ and PbTatD-D352A strains exhibited a twofold longer survival time.

a

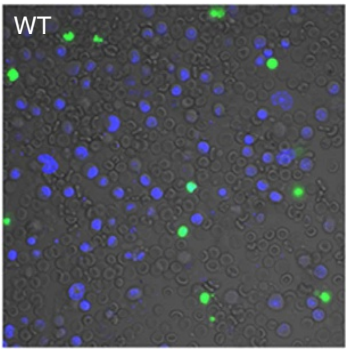

b

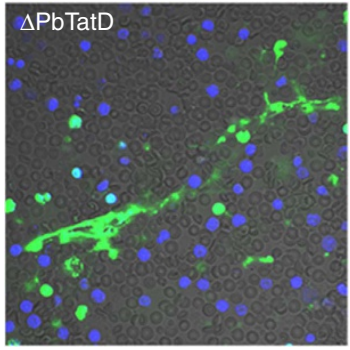

d

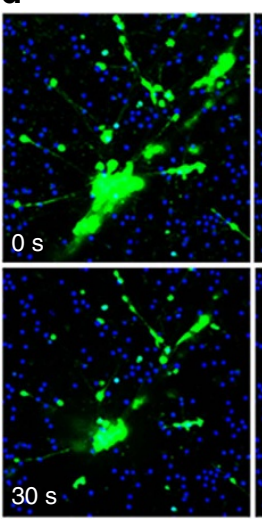

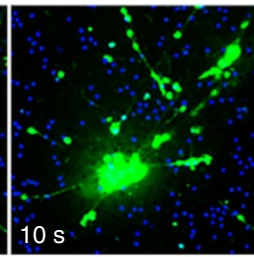

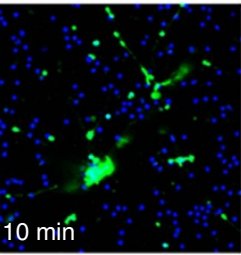

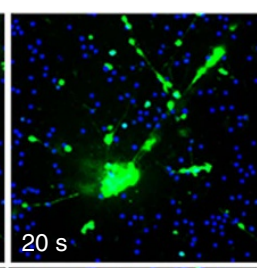

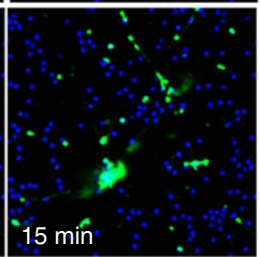

C

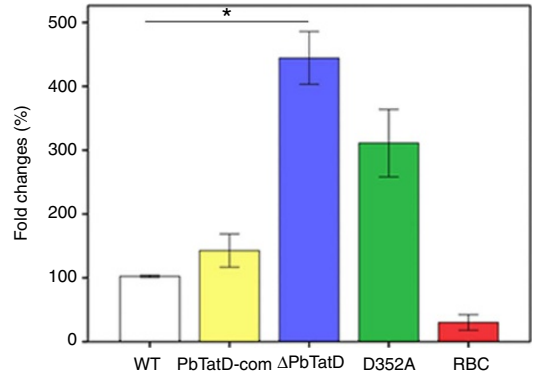

e
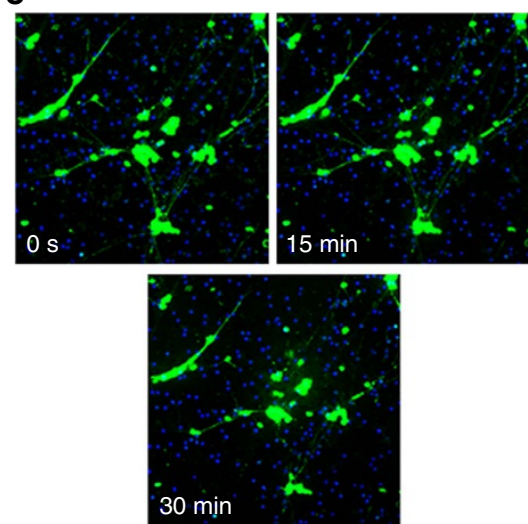

Figure 5 | Formation of ETs after incubation of infected erythrocytes with macrophages. Representative images of the extracellular network structures after incubation of J774A.1 macrophages with WT P. berghei (a) and $\triangle$ PbTatD (b) infected erythrocytes. The ETs and dead cell nuclei were stained with Sytox green. Scale bar, $20 \mu \mathrm{m}$. (c) The relative quantification of ETs released by J774A.1 macrophages stimulated with the WT strain (white bar), $\Delta \mathrm{PbTatD}$ strain (blue bar), PbTatD-com strain (yellow bar), PbTatD-D352A strain (green bar) or RBCs (red bar) are shown. The WT group was regarded as 100\%. The results are the averages of three independent experiments (mean \pm s.e.m., ${ }^{\star} P<0.05$ in two-tailed Student's $t$-test). (d,e) Images of ETs after the addition of rPbTatD-GST or rPbTatD-D352A-GST in the cultivation at various time points. rPbTatD-GST showed DNase activity in a time-dependent manner (d), whereas rPbTatD-D352A-GST did not show any activity (e).

In this study, a gene encoding the E. coli TatD-like homologous protein was identified in every genome of plasmodial parasites. Bioinformatics analysis showed that the TatD sequences of Plasmodium, including $P$. falciparum, P. vivax, P. knowlesi, $P$. chabaudi, $P$. yoelii and $P$. berghei, shared more than $80 \%$ identity, which indicates a conserved function for this protein in the Plasmodium genus (Fig. 1). All plasmodial TatD sequences contained a conserved motif with the 21 AA sequence characteristic of the TatD deoxyribonuclease family ${ }^{22}$. In addition to the conserved deoxyribonuclease motif, a signal 


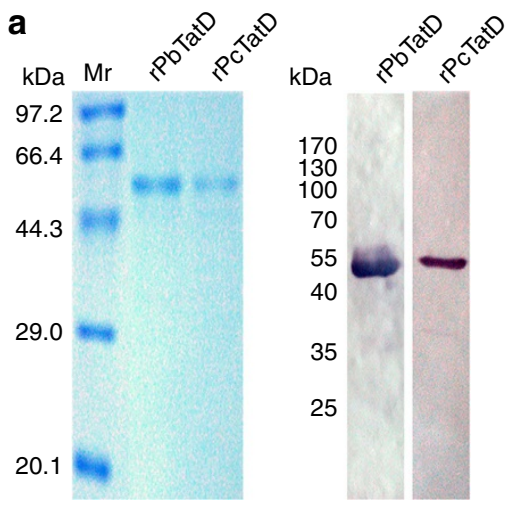

b

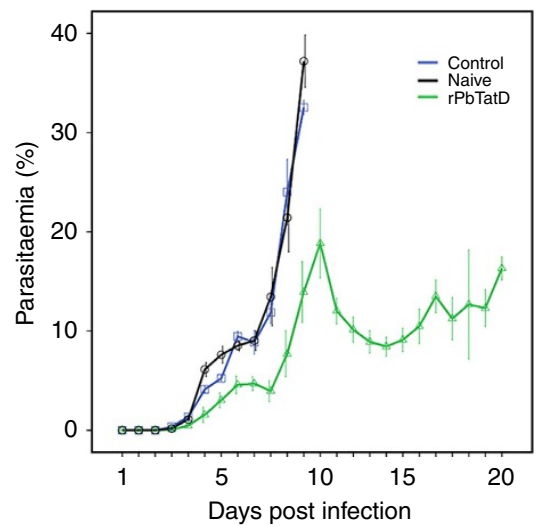

e

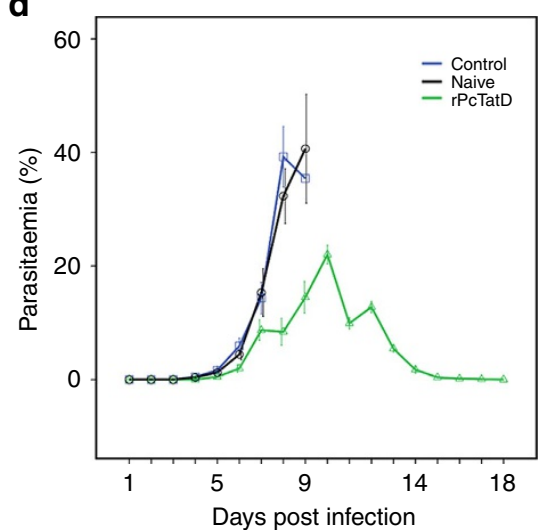

g

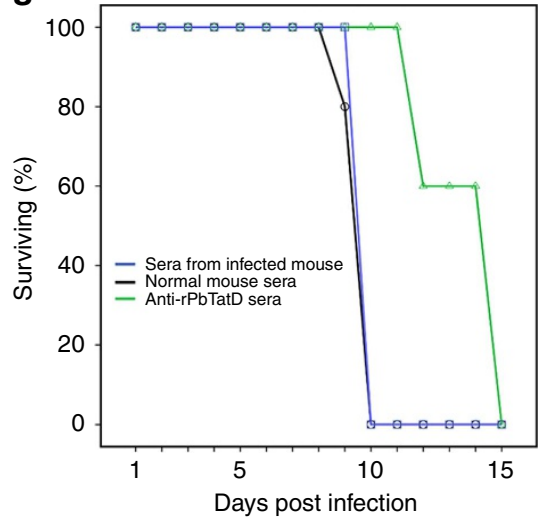

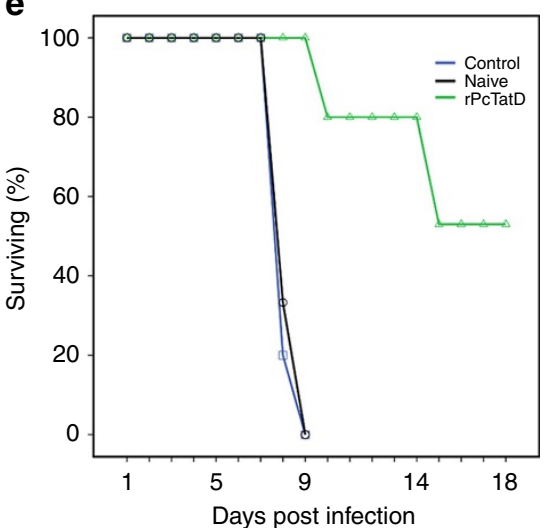

h

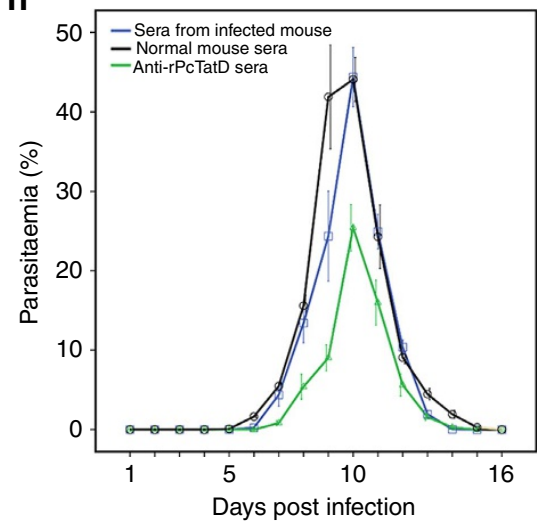

c

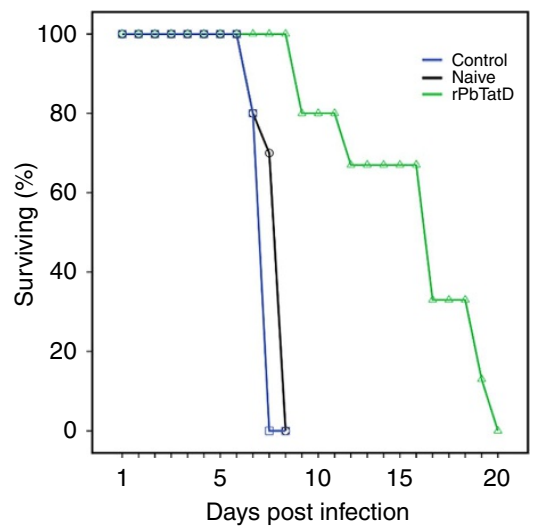

f

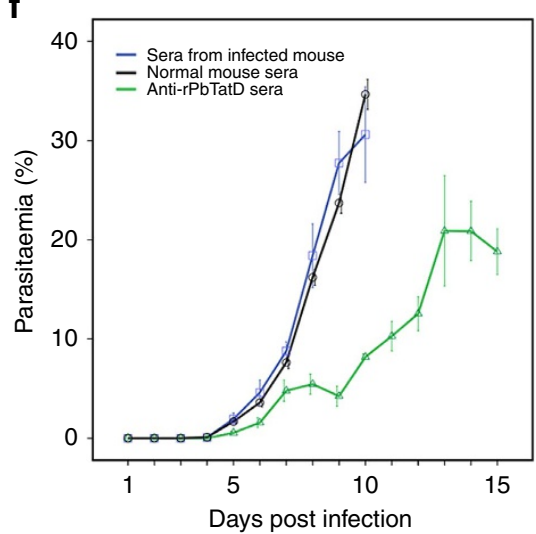

i

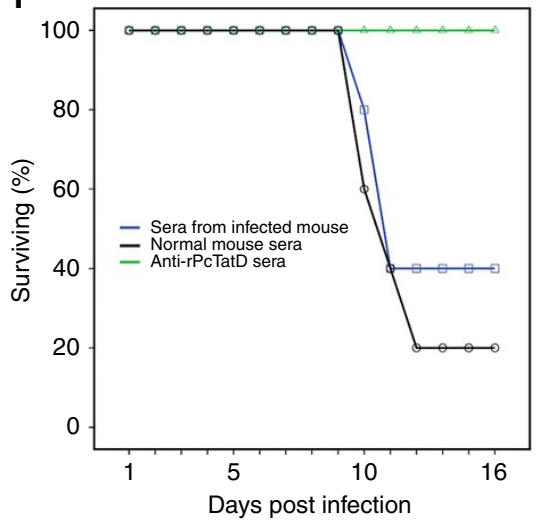

Figure 6 | Immunization with the TatD-like DNase generates protective immunity against rodent malaria. (a) rPbTatD-HIS and rPcTatD-HIS were purified by affinity chromatography using His GraviTrap. The SDS-PAGE and western blotting assays revealed that the recombinant proteins were $>90 \%$ pure. (b) BALB/c mice immunized with Freund's adjuvant alone or without any immunization (Naive) exhibited 3.12-fold higher parasitaemia than the rPbTatD-immunized group on day 11 post infection; the error bars indicate the s.d. (c) Mice in the rPbTatD-immunized group survived 10 days longer than the two control groups. (d,e) BALB/c mice immunized with Freund's adjuvant alone and naive mice exhibited 4.66-fold higher parasitaemia than the rPcTatD-immunized group on day 8 post challenge; the error bars indicate the s.d. All of the mice that received Freund's adjuvant alone and the naive group died on day 9 post infection; however, eight of the mice in the rPbTatD-immunized group were completely protected. (f,g) The group that received serum from a previously infected mouse exhibited 3.97-fold higher parasitaemia than the group that received anti-rPbTatD serum on day 10 post infection. Compared with the other groups, the group that received anti-rPbTatD serum survived for 5 days longer; the error bars indicate the s.d. (h,i) The group that received serum from a previously infected mouse exhibited 1.79 -fold higher parasitaemia than the group that received rPcTatD-specific serum on day 10 post infection. The mice that received rPcTatD-specific serum showed a $100 \%$ survival rate. The error bars indicate the s.d.

peptide was exclusively found in the $\mathrm{N}$ termini of all of Plasmodium TatD proteins and was absent in other apicomplexan parasites and metazoans (Fig. 1). The indirect immunofluorescence and electron microscopy results demonstrated that TatD-like DNase is expressed in the blood stage of plasmodial parasites and is likely secreted during parasite maturation. The electron microscopy results showed that the protein is transported towards the erythrocyte membrane, which 
is consistent with the bioinformatics analysis. PfTatD does not contain the Pexel motif ${ }^{28}$ frequently identified in the membrane protein, which indicated that this class of proteins follows the classical pathway of transportation.

The possible association of TatD expression with parasite virulence was investigated by various approaches, including real-time PCR (rtPCR), western blotting, gene knockout and complementation. The expression of TatD was increased in the virulent $P$. bergeri ANKA strain compared with the avirulent strain ( $P$. bergeri NK65). Similar data were obtained when TatD expression was assessed in the P. yoelii strains (Fig. 3). Using recombinant proteins, we confirmed that plasmodial TatD is critical for DNA hydrolysis. Unlike other DNases, such as DNase II, the DNA hydrolytic activity of plasmodial TatD is not divalent metal-dependent (Supplementary Fig. 7).

It has been well established that that host macrophages and neutrophils release ETs to confine invading pathogens on activation. Some of the pathogens release DNase to counteract the trap. The trap structure has been observed in skin blood vessels of children with uncomplicated malaria ${ }^{14}$. Furthermore, Toxoplasma gondii, another Apicomplexan, can induce neutrophils to release NETs directly ${ }^{29}$. In the current experiment, only the TatD knockout and TatD mutated parasites, and not the WT strain, were able to induce ET. In addition, ETs were immediately destroyed on addition of recombinant TatD to the culture. Thus, the data support the conclusion that plasmodial parasites use TatD to degrade ET structures to escape host immune clearance.

The sequence conservation and significant DNA hydrolytic activity of plasmodial TatD led us to further explore the possibility of using this molecule as an immunogen for vaccination. Surprisingly, we observed an increase in protection in two mouse models (both C57BL/6 and BALB/c) (Fig. 6; Supplementary Figs. 10 and 11). These data indicate that the plasmodial TatD protein should be further explored as a potential candidate in malaria vaccine development.

In conclusion, we identified a TatD-like DNase that is present in every Plasmodium species and has biochemical features distinct from those of the bacterial TatD family. The enzyme was predominantly expressed in mature asexual-stage parasites but may also be expressed at other stages ${ }^{30}$. The expression of this enzyme was associated with parasite virulence and was necessary for successful proliferation in the host. Moreover, antibodies against plasmodial TatD provided protection against parasite infection, which supports the use of $P$. falciparum TatD-like DNase as a potential candidate for malarial vaccine development.

\section{Methods}

Parasites. The P. falciparum 3D7 strain was cultured and synchronized in vitro as previously described ${ }^{31}$. Briefly, parasites were continuously cultured in MCM culture medium in candle gar and incubated at $37^{\circ} \mathrm{C}$. The synchronization of parasites was performed by incubating the cultures in $5 \%(\mathrm{w} / \mathrm{v})$ D-sorbitol (Sigma, USA), after which the cells were washed twice with MCM before transferring to new flasks.

P. berghei (strains ANKA and NK65), P. chabaudi (strain AS) and P. yoeli (strains YM, XNL, ING and 17XL) were maintained through infection of C57BL/6 mice by IP injection of $1 \times 10^{6}$ parasitized erythrocytes as previously described ${ }^{32}$. Parasitaemia was monitored by examining Giemsa-stained thin blood smears under a light microscope.

Six- to eight-week-old BALB/c and C57BL/6 female mice were purchased from Jilin University Experimental Animal Center (Changchun, China). All mice were maintained in an enriched environment, and all experiments were carried out in accordance with the guidelines established by Jilin University and were approved by the Animal Welfare and Research Ethics Committee of Jilin University. The ethical clearance number for this study is KZ2010-01.

Plasmodium TatD-like DNase sequence analyses. The putative TatD nuclease sequences of $P$. falciparum, $P$. berghei, P. knowlesi, P. vivax, P. chabaudi, P. yoelii, Babesia equi, Cryptosporidium muris, Trypanosoma brucei, Theileria parva,
Neospora caninum, Leishmania, Homo sapiens, E. coli and Schistosoma mansoni were compared by a multiple alignment constructed with DNAMAN 7 (Lynnon Corporation, USA). To assess the extent of the diversity and evolutionary relationships among the TatD nucleases (Gene IDs for phylogenies and comparisons are shown in Supplementary Table 1), the MEGA 5.2 program $^{33}$ was used to construct a phylogenetic tree on the basis of the evolutionary distances calculated by the Kimura two-parameter model ${ }^{34}$

The putative function of PfTatD was identified by GO analysis (http://geneontology.org/). The signal peptide was predicted using the SignalP 4.1 server $^{35}$. The PfTatD 3D model was built using SWISS-MODEL ${ }^{36-38}$.

Total RNA extraction and quantitative rtPCR. Total RNA was extracted from synchronized parasites every $8 \mathrm{~h}$ throughout the entire life cycle as previously described $^{39}$. The rodent parasites were collected at a mixed stage when parasitaemia reached $\sim 5 \%$. rtPCR was performed in triplicate using the FastStart Universal SYBR Green Master (Roche) with specific primers (Supplementary Table 2) and an Applied Biosystems 7500 rtPCR system. The data were analysed using the comparative critical threshold $(\Delta \Delta \mathrm{Ct})$ method, in which the amount of target RNA was compared to an internal control, seryl-tRNA synthetase (PF3D7_1205100) ${ }^{40}$. Rodent malarial TatD transcription was evaluated using the same method.

Expression of recombinant proteins and antibody generation. Genes encoding the TatD-like DNase of $P$. falciparum, $P$. berghei and $P$. chabaudi were cloned from the corresponding parasites using the rtPCR method. The sequences coding for the H303 and D352 mutations in PbTatD were chemically synthesized, and all genes were cloned into the pET-28a and pGEX-4T-1 vectors, respectively (Invitrogen). HIS-tagged and GST-tagged recombinant proteins were expressed in BL21(DE3) cells using the pET-28a and pGEX-4T-1 vectors, respectively, and the soluble proteins were purified using His GraviTrap and Glutathione Sepharose (GE Healthcare), respectively, according to the manufacturer's instructions. Anti-rPfTatD/rPbTatD/rPcTatD sera were raised in New Zealand white rabbits by four immunizations with $300 \mu \mathrm{g}$ of recombinant protein and Freund's adjuvant. The sera were collected following titre evaluation. Specific IgGs were purified from the sera using Protein A (GE Healthcare) according to the manufacturer's instructions.

Characterization of DNase activity in recombinant PfTatD. Human wholeblood cells were obtained from the blood bank of Changchun City, and the experiments with human genetic samples were approved by the Ethical Committee of Jilin University (the ethical clearance number for this study is KZ2010-01). Genomic DNA was extracted from whole-blood cells using the QIAamp DNA Mini Kit (Qiagen) according to the manufacturer's protocol. The DNA was incubated with $30,15,7.5,3$ or $1.5 \mu \mathrm{M}$ recombinant PfTatD in Tris- $\mathrm{HCl}$ buffer ( $\mathrm{pH} 7.5$ ) in a final volume of $40 \mu \mathrm{l}$ at $37^{\circ} \mathrm{C}$. To search for the optimal enzymatic temperature, digestion reactions were performed at $16,25,35,37$ and $41^{\circ} \mathrm{C}$. To determine the effect of metallic ions on nuclease activity, different concentrations of EDTA, $\mathrm{Mg}^{2+}, \mathrm{Ni}^{2+}$ and other bivalent metal ions were added to the reaction. The results were visualized on $1.2 \%$ agarose gels under UV illumination following ethidium bromide staining.

Parasite protein preparation and western blotting analysis. The proteins from synchronized $P$. falciparum-iRBCs were run on an SDS-PAGE gel and analysed via western blotting. Briefly, the proteins were electrophoresed on $12 \%$ SDS-PAGE gels and then transferred to $0.2-\mu \mathrm{m}$ nitrocellulose membranes using the Semi-Dry blotting system (Bio-Rad, CA, USA). A rabbit polyclonal anti-rPfTatD $(1: 1,000)$ IgG was used as the primary antibody, and either an AP-labelled goat anti-rabbit IgG $(1: 20,000)$ (Abcam, Shanghai, China) or an HRP-labelled goat anti-rabbit IgG $(1: 6,000)$ (Abcam, Shanghai, China) was used as the secondary antibody. Rodent parasite-infected erythrocytes were collected at 5\% parasitaemia for every strain. The samples were analysed as described above with the corresponding specific primary antibody. The host proteins used in the cross-reactivity assay were prepared by grinding them with liquid nitrogen, and the primary antibody used was a mouse anti-rPbTatD IgG. A GAPDH monoclonal antibody (Abcam) and rabbit anti-HSP70 polyclonal antibodies (Antibodies-online, Germany) were used in a corresponding experiment, and either the AP-labelled goat anti-mouse IgG $(1: 20,000)$ or the HRP-labelled goat anti-rabbit IgG $(1: 5,000)$ was used as the secondary antibody. Full versions of all blots are provided in Supplementary Fig. 13.

Immunofluorescence assay. The air-dried thin smears of blood cells infected with parasites at different stages were fixed with $4 \%$ paraformaldehyde and $0.0025 \%$ glutaraldehyde for $30 \mathrm{~min}$ at room temperature and were then blocked with $5 \%$ non-fat milk. The samples were incubated with a rabbit anti-TatD-specific IgG (1:100) for $60 \mathrm{~min}$ at $37^{\circ} \mathrm{C}$, followed by staining with a goat anti-rabbit IgG (1:600) (Alexa Fluor 488) for $60 \mathrm{~min}$ at $37^{\circ} \mathrm{C}$. The nuclei were stained with 4,6-diamidino2-phenylindole (DAPI). The images were captured using a fluorescence microscope (Olympus, BX 53). 
Immunoelectron microscopy assay. For immunoelectron microscopy assay, mature trophozoite-infected erythrocytes ( $P$. falciparum 3D7 and $P$. berghei ANKA) were fixed with $0.2 \% \mathrm{v} / \mathrm{v}$ glutaraldehyde and $1 \% \mathrm{w} / \mathrm{v}$ paraformaldehyde in PBS for $30 \mathrm{~min}$ at $4{ }^{\circ} \mathrm{C}$. The samples were embedded in LR white resin (Sigma) at $50{ }^{\circ} \mathrm{C}$ for $24 \mathrm{~h}$ after dehydration. Thin sections were mounted on nickel grids. For immunostaining, samples were incubated with a TatD-like DNase-specific rabbit polyclonal IgG $(1: 100)$ at $4{ }^{\circ} \mathrm{C}$ overnight after blocking with $3 \%$ non-fat milk, followed by incubation with a goat anti-rabbit IgG conjugated to $5 \mathrm{~nm}$ gold (1:40) (Sigma G7277) at $37^{\circ} \mathrm{C}$ for $60 \mathrm{~min}$. The IgG from pre-immune sera was used as the primary antibody control. The samples were examined with a transmission electron microscope (Hitachi H-7650, Japan).

DNA constructs and parasite transfection. We chose the double-crossover method to generate a TatD knockout as previously described ${ }^{41}$. Briefly, as presented in Supplementary Fig. 3a, the plasmid was built with pl0001 (MR4, ATCC, USA). Two homology sequences from the PbTatD $5^{\prime}$ and $3^{\prime}$ Untranslated regions (UTRs) were PCR-amplified and inserted upstream and downstream of the DHFR gene, respectively. A total of $20 \mu \mathrm{g}$ of linear plasmid (digested with ApaI and NotI) was mixed with $10^{7}$ schizonts purified with $55 \%$ Nycodenz (AXIS-SHIELD). The mixture was pulsed at $0.8 \mathrm{kV}$ and a capacitance of $25 \mu \mathrm{F}$ using a Gene Pulser II (Bio-Rad) and then IV injected into one C57BL/6 mouse. The parasites were selected in vivo by the addition of a pyrimethamine solution to the drinking water $24 \mathrm{~h}$ after injection. A PCR method with primers specific for the insert was used to verify the transfection and the gene knockout The PbTatD knockout strain parasites $(\triangle \mathrm{PbTatD})$ were cloned using a limitingdilution method.

To generate a parasite strain with a reconstructed TatD gene in the $\triangle \mathrm{PbTatD}$ strain, the plasmid pL0017 (MR4, ATCC, USA) was used as a backbone for the generation of the PbTatD complementation construct and to target the c- or d-ssu-rrna sequence for integration by single crossover recombination. Briefly, the hDHFR selection marker was amplified from a PYC plasmid (a gift from Professor Jing Yuan, Ximen University) and replaced the GFPM3 (BamHI/XbaI) in pL0017 to build pL0017-hDHFR. Then, the PbTatD-containing $3^{\prime}$ UTR was amplified from the genome and exchanged the GFPM3 (BamHI/XbaI) in a new pL0017 backbone to build pL0017-TatD. Pbeeflaa + PbTatD + 3' UTR was amplified from pL0017TatD and replaced the DHFR-TS cassette (HindIII/EcoRV) in pL0017-hDHFR to obtain the PbTatD complementation plasmid (PbTatD-com). The PbTatD-com plasmid was linearized with SacII and transfected into the $\triangle \mathrm{PbTatD}$ parasites as previously described. The transfected parasites were selected after a 4-day-IV treatment with the drug WR99210 (Sigma). The correct integration of the complementation construct into the d-ssu-rrna on chromosome 5 was verified by PCR using specific primers (Supplementary Table 2). Expression of the PbTatD gene in PbTatD-com parasites was analysed by western blot analysis using an anti-rPbTatD IgG as the primary antibody and glyceraldehyde-3-phosphate dehydrogenase (GAPDH) as the control.

To generate a parasite strain with a mutation at the 352 position, we used the pl0001 plasmid to generate the allelic replacement construct of PbTatD-D352A. The fragment $(-1,500$ to $-1,000 \mathrm{bp})$ was amplified and inserted at the $5^{\prime}$ end of the DHFR-TS cassette. The fragment $(-1,000$ to $+1,389$ bp with mutation) was chemically synthesized by Sangon Biotech (Shanghai Co., Ltd) and was inserted at the $3^{\prime}$ end of the DHFR-TS cassette, as illustrated in Supplementary Fig. 5. The sequences of the primers used for this study are listed in Supplementary Table 2.

ET formation assay in vitro. J774A.1 macrophages were purchased from the Cell Resource Center, IBMS, China and were cultivated in DMEM on poly-L-lysinecoated glass-bottom dishes with a confluence of $1 \times 10^{5}$ cells/well. A total of $10^{6}$ mouse red blood cells (5\% parasitaemia) infected with either the WT P. berghei ANKA or $\triangle \mathrm{PbTatD}$ strain were added to the dishes and cultivated for $6 \mathrm{~h}$. A total of $10^{6}$ normal mouse red blood cells were used as the control. After co-cultivation, the medium was removed, and the cells were stained with Sytox green (a cellimpermeable dye) and DAPI (a cell-permeable dye) for $5 \mathrm{~min}$ without fixation and then imaged immediately for the presence of NETs via confocal laser microscopy. In some of the experiments, rPbTatD-D352A-GST and rPbTatD-GST were added after staining. ETs were counted in 50 fields when the length of extracellular DNA was over $50 \mu \mathrm{m}$. Three independent experiments were performed.

Mouse neutrophils were purified using Percoll as previously described ${ }^{29}$. Briefly, mouse blood was collected, washed and resuspended in RPMI. The cells were layered onto a three-layer Percoll gradient of 55, 65 and 80\% Percoll and centrifuged at $500 \mathrm{~g}$ for $30 \mathrm{~min}$ at $4{ }^{\circ} \mathrm{C}$. The neutrophils $(65 / 80 \%$ interface) were collected, washed twice with PBS, resuspended in RPMI and counted. The neutrophils were incubated with different $P$. berghei ANKA strains for $2 \mathrm{~h}$.

Immunization with rPbTatD and rPcTatD proteins. For the first immunization, $50 \mu \mathrm{g} / \mathrm{mouse}$ (BALB/c or C57BL/6 strain, female, six- to eight-week-old) rPbTatDHIS or rPcTatD-HIS emulsified with complete Freund's adjuvant was intramuscular injected, followed by three immunizations with $50 \mu \mathrm{g}$ recombinant protein emulsified with incomplete Freund's adjuvant with 2 -week inter-injection intervals. The mice in the control group were injected with Freund's adjuvant only. The antibody titres were evaluated by ELISA. When the titre exceeded 1:32,000, $\sim 10^{6}$
iRBCs per mouse were IP injected in all groups. The parasitaemia was measured daily by counting 3,000 cells per Giemsa-stained blood smear.

Passive immunization assays with anti-rPbTatD/rPcTatD sera. In the passive immunization assays, each mouse was IV injected with $500 \mu \mathrm{l}$ of sera from either an $\mathrm{rPbTatD} / \mathrm{rPcTatD}$-immunized mouse or a mouse previously infected with $P$. berghei ANKA or P. chabaudi AS $24 \mathrm{~h}$ earlier. After challenging with $10^{5}$ iRBCs, the parasitaemia was measured and counted as described above.

\section{References}

1. von Kockritz-Blickwede, M. et al. Phagocytosis-independent antimicrobial activity of mast cells by means of extracellular trap formation. Blood 111, 3070-3080 (2008).

2. Yousefi, S. et al. Catapult-like release of mitochondrial DNA by eosinophils contributes to antibacterial defense. Nat. Med. 14, 949-953 (2008).

3. Brinkmann, V. et al. Neutrophil extracellular traps kill bacteria. Science 303, 1532-1535 (2004).

4. Urban, C. F. et al. Neutrophil extracellular traps contain calprotectin, a cytosolic protein complex involved in host defense against Candida albicans. PLoS Pathog. 5, e1000639 (2009).

5. Buchanan, J. T. et al. DNase expression allows the pathogen group A Streptococcus to escape killing in neutrophil extracellular traps. Curr. Biol. 16, 396-400 (2006).

6. Walker, M. J. et al. DNase Sdal provides selection pressure for a switch to invasive group A streptococcal infection. Nat. Med. 13, 981-985 (2007).

7. Harding, M. \& Kubes, P. Innate immunity in the vasculature: interactions with pathogenic bacteria. Curr. Opin. Microbiol. 15, 85-91 (2012).

8. Berends, E. T. et al. Nuclease expression by Staphylococcus aureus facilitates escape from neutrophil extracellular traps. J. Innate Immun. 2, 576-586 (2010).

9. Thammavongsa, V., Missiakas, D. M. \& Schneewind, O. Staphylococcus aureus degrades neutrophil extracellular traps to promote immune cell death. Science 342, 863-866 (2013).

10. Seper, A. et al. Vibrio cholerae evades neutrophil extracellular traps by the activity of two extracellular nucleases. PLoS Pathog. 9, e1003614 (2013).

11. Chen, G. et al. Heme-induced neutrophil extracellular traps contribute to the pathogenesis of sickle cell disease. Blood 123, 3818-3827 (2014).

12. Ferreira, A., Balla, J., Jeney, V., Balla, G. \& Soares, M. P. A central role for free heme in the pathogenesis of severe malaria: the missing link? J. Mol. Med. 86, 1097-1111 (2008).

13. Pamplona, A. et al. Heme oxygenase- 1 and carbon monoxide suppress the pathogenesis of experimental cerebral malaria. Nat. Med. 13, 703-710 (2007)

14. Baker, V. S. et al. Cytokine-associated neutrophil extracellular traps and antinuclear antibodies in Plasmodium falciparum infected children under six years of age. Malar. J. 7, 41 (2008).

15. Miller, L. H., Good, M. F. \& Milon, G. Malaria pathogenesis. Science 264, 1878-1883 (1994).

16. Scherf, A., Lopez-Rubio, J. J. \& Riviere, L. Antigenic variation in Plasmodium falciparum. Annu. Rev. Microbiol. 62, 445-470 (2008).

17. Deitsch, K. W., Lukehart, S. A. \& Stringer, J. R. Common strategies for antigenic variation by bacterial, fungal and protozoan pathogens. Nat. Rev. Microbiol. 7, 493-503 (2009).

18. Matos, C. F., Di Cola, A. \& Robinson, C. TatD is a central component of a Tat translocon-initiated quality control system for exported FeS proteins in Escherichia coli. EMBO Rep. 10, 474-479 (2009).

19. Chen, L. et al. Phytophthora sojae TatD nuclease positively regulates sporulation and negatively regulates pathogenesis. Mol. Plant Microbe Interact 27, 1070-1080 (2014).

20. Nagano, N., Orengo, C. A. \& Thornton, J. M. One fold with many functions: the evolutionary relationships between TIM barrel families based on their sequences, structures and functions. J. Mol. Biol. 321, 741-765 (2002).

21. de Koning-Ward, T. F., Janse, C. J. \& Waters, A. P. The development of genetic tools for dissecting the biology of malaria parasites. Annu. Rev. Microbiol. 54, 157-185 (2000).

22. Wexler, M. et al. TatD is a cytoplasmic protein with DNase activity. No requirement for TatD family proteins in sec-independent protein export. J. Biol. Chem. 275, 16717-16722 (2000).

23. Qiu, J., Yoon, J. H. \& Shen, B. Search for apoptotic nucleases in yeast: role of Tat-D nuclease in apoptotic DNA degradation. J. Biol. Chem. 280, 1537015379 (2005).

24. Chen, Y. C., Li, C. L., Hsiao, Y. Y., Duh, Y. \& Yuan, H. S. Structure and function of TatD exonuclease in DNA repair. Nucleic Acids Res. 42, 10776-10785 (2014).

25. Gannavaram, S. \& Debrabant, A. Involvement of TatD nuclease during programmed cell death in the protozoan parasite Trypanosoma brucei. Mol. Microbiol. 83, 926-935 (2012).

26. Derre-Bobillot, A. et al. Nuclease A (Gbs0661), an extracellular nuclease of Streptococcus agalactiae, attacks the neutrophil extracellular traps and is needed for full virulence. Mol. Microbiol. 89, 518-531 (2013). 
27. Stevenson, M. M. \& Riley, E. M. Innate immunity to malaria. Nat. Rev. Immunol. 4, 169-180 (2004).

28. Marti, M., Good, R. T., Rug, M., Knuepfer, E. \& Cowman, A. F. Targeting malaria virulence and remodeling proteins to the host erythrocyte. Science 306, 1930-1933 (2004).

29. Abi Abdallah, D. S. et al. Toxoplasma gondii triggers release of human and mouse neutrophil extracellular traps. Infect. Immun. 80, 768-777 (2012).

30. Silvestrini, F. et al. Protein export marks the early phase of gametocytogenesis of the human malaria parasite Plasmodium falciparum. Mol. Cell. Proteomics 9, 1437-1448 (2010).

31. Trager, W. \& Jensen, J. B. Human malaria parasites in continuous culture. Science 193, 673-675 (1976).

32. Sanni, L. A., Fonseca, L. F. \& Langhorne, J. Mouse models for erythrocytic-stage malaria. Methods Mol. Med. 72, 57-76 (2002).

33. Tamura, K., Dudley, J., Nei, M. \& Kumar, S. MEGA4: molecular evolutionary genetics analysis (MEGA) software version 4.0. Mol. Biol. Evol. 24, 1596-1599 (2007).

34. Saitou, N. \& Nei, M. The neighbor-joining method: a new method for reconstructing phylogenetic trees. Mol. Biol. Evol. 4, 406-425 (1987).

35. Petersen, T. N., Brunak, S., von Heijne, G. \& Nielsen, H. SignalP 4.0: discriminating signal peptides from transmembrane regions. Nat. Methods $\mathbf{8}$, 785-786 (2011)

36. Arnold, K., Bordoli, L., Kopp, J. \& Schwede, T. The SWISS-MODEL workspace: a web-based environment for protein structure homology modelling. Bioinformatics 22, 195-201 (2006).

37. Biasini, M. et al. SWISS-MODEL: modelling protein tertiary and quaternary structure using evolutionary information. Nucleic Acids Res. 42, W252-W258 (2014).

38. Benkert, P., Biasini, M. \& Schwede, T. Toward the estimation of the absolute quality of individual protein structure models. Bioinformatics 27, 343-350 (2011).

39. Kyes, S., Pinches, R. \& Newbold, C. A simple RNA analysis method shows var and rif multigene family expression patterns in Plasmodium falciparum. Mol. Biochem. Parasitol. 105, 311-315 (2000).

40. Moll, K. et al. A novel DBL-domain of the P. falciparum 332 molecule possibly involved in erythrocyte adhesion. PloS ONE 2, e477 (2007).
41. Janse, C. J., Ramesar, J. \& Waters, A. P. High-efficiency transfection and drug selection of genetically transformed blood stages of the rodent malaria parasite Plasmodium berghei. Nat. Protoc. 1, 346-356 (2006).

\section{Acknowledgements}

We sincerely appreciate the technical assistance of the technicians at the Core Facility of the Institute of Zoonosis, Jilin University. This study was supported by the National Natural Science Foundation of China $(81130033,81171592)$ and the Jilin provincial grants (2015Y033 and 20150204020NY).

\section{Author contributions}

Z.C., N.J., M.W. and Q.C. conceived and designed the study. Z.C. and Q.C. wrote the manuscript. Y.Z., H.L. and J.Y. performed the study. X.C. and Y.C. provided research protocols and reagents. All of the authors have read and agreed with the data.

\section{Additional information}

Supplementary Information accompanies this paper at http://www.nature.com/ naturecommunications

Competing financial interests: The authors declare no competing financial interests.

Reprints and permission information is available online at http://npg.nature.com/ reprintsandpermissions/

How to cite this article: Chang, Z. et al. The TatD-like DNase of Plasmodium is a virulence factor and a potential malaria vaccine candidate. Nat. Commun. 7:11537 doi: $10.1038 /$ ncomms11537 (2016)

\section{(c) (i)}

This work is licensed under a Creative Commons Attribution 4.0 International License. The images or other third party material in this article are included in the article's Creative Commons license, unless indicated otherwise in the credit line; if the material is not included under the Creative Commons license users will need to obtain permission from the license holder to reproduce the material. To view a copy of this license, visit http://creativecommons.org/licenses/by/4.0/ 